\title{
Recent Symbolic Summation Methods to Solve Coupled Systems of Differential and Difference Equations
}

\author{
Carsten Schneider*t \\ Research Institute for Symbolic Computation (RISC) \\ Johannes Kepler University, Altenbergerstraße 69, A-4040 Linz, Austria \\ E-mail: Carsten.Schneidererisc.jku.at

\section{Johannes Blümlein and Abilio de Freitas} \\ Deutsches Elektronen-Synchrotron, DESY, \\ Platanenallee 6, D-15738 Zeuthen, Germany \\ E-mail: johannes.bluemlein@desy.de, abilio.de.freitas@desy.de
}

\begin{abstract}
We outline a new algorithm to solve coupled systems of differential equations in one continuous variable $x$ (resp. coupled difference equations in one discrete variable $N$ ) depending on a small parameter $\varepsilon$ : given such a system and given sufficiently many initial values, we can determine the first coefficients of the Laurent-series solutions in $\varepsilon$ if they are expressible in terms of indefinite nested sums and products. This systematic approach is based on symbolic summation algorithms in the context of difference rings/fields and uncoupling algorithms. The proposed method gives rise to new interesting applications in connection with integration by parts (IBP) methods. As an illustrative example, we will demonstrate how one can calculate the $\varepsilon$-expansion of a ladder graph with 6 massive fermion lines.
\end{abstract}

Loops and Legs in Quantum Field Theory - LL 2014,

27 April - 2 May 2014

Weimar, Germany

\footnotetext{
*Speaker.

$\dagger$ This work was supported in part by DFG Sonderforschungsbereich Transregio 9, Computergestützte Theoretische Teilchenphysik, the Austrian Science Fund (FWF) grants P20347-N18 and SFB F50 (F5009-N15), and the European Commission through contract PITN-GA-2010-264564 (LHCPhenoNet) and PITN-GA-2012-316704 (HIGGSTOOLS).
} 


\section{Introduction}

Symbolic summation in the setting of difference fields and rings $[26,32,20,33,35,36,37$, 38, 40] provides a general toolbox in form of the Mathematica package Sigma [34, 39] to simplify definite multi-sums to expressions in terms of indefinite nested sums and products. This function domain covers as special cases harmonic sums [16, 46]

$$
S_{a_{1}, \ldots, a_{k}}(N)=\sum_{i_{1}=1}^{N} \frac{\operatorname{sign}\left(a_{1}\right)^{i_{1}}}{i_{1}^{\left|a_{1}\right|}} \sum_{i_{2}=1}^{i_{1}} \frac{\operatorname{sign}\left(a_{2}\right)^{i_{2}}}{i_{2}^{\left|a_{2}\right|}} \ldots \sum_{i_{k}=1}^{i_{k-1}} \frac{\operatorname{sign}\left(a_{k}\right)^{i_{k}}}{i_{k}^{\left|a_{k}\right|}}, \quad a_{i} \in \mathbb{Z} \backslash\{0\},
$$

generalized harmonic sums [30, 7], cyclotomic sums [3] or nested binomial sums [24, 23, 47, 11] .

In the last years this technology proved to be useful to evaluate non-trivial Feynman integrals in the context of QCD. Namely, as worked out in [17, 48] a big class of integrals in terms of the dimensional parameter $\varepsilon=D-4$ and a discrete Mellin parameter $N$ can be written in the following form:

$$
F(N)=\sum_{k_{1}=l_{1}}^{L_{1}(N)} \ldots \sum_{k_{v}=l_{v}}^{L_{v}\left(N, k_{1}, \ldots, k_{v-1}\right)} f\left(\varepsilon, N, k_{1}, \ldots, k_{v}\right)
$$

where $L_{i}\left(N, k_{1}, \ldots, k_{v-1}\right)$ stands for an integer linear relation in the variables $N, k_{1}, \ldots, k_{v-1}$ or is $\infty$ and $f\left(\varepsilon, N, k_{1}, \ldots, k_{v}\right)$ is a linear combination of proper hypergeometric sequences given in terms of $\Gamma$-functions with arguments in terms of integer linear relation in the integer parameters $N, k_{1}, \ldots, k_{v-1}$ and $\varepsilon$ might occur linearly in the form $r \varepsilon$ with $r$ being a rational number.

Given such a multi-sum $F(N)$, the main task is to compute the first coefficients $F_{i}(N)$ of the Laurent-series expansion

$$
F(N) \stackrel{?}{=} F_{\lambda}(N) \varepsilon^{\lambda}+F_{\lambda+1}(N) \varepsilon^{\lambda+1}+\ldots, \quad \lambda \in \mathbb{Z}
$$

in terms of special functions such as (generalized) (cyclotomic) harmonic sums and nested binomial sums mentioned above. To get this representation, the following two tactics are of interest.

Tactic 1: Compute the coefficients of the $\varepsilon$-expansion of the summand

$$
f\left(\varepsilon, N, k_{1}, \ldots, k_{v}\right)=f_{\lambda}\left(N, k_{1}, \ldots, k_{v}\right) \varepsilon^{\lambda}+f_{\lambda+1}\left(N, k_{1}, \ldots, k_{v}\right) \varepsilon^{\lambda+1} \ldots
$$

by formulas such as Eq. (1.4) in [5] and arrive at a linear combination of hypergeometric terms (free of $\varepsilon$ ) multiplied with (cyclotomic) harmonic sums. Finally, if the interchange of the summation signs and differentiation w.r.t. $\varepsilon$ (which we applied to get the summand expansion) is valid, we end up at multi-sum representations for the coefficients of the expansion (1.3):

$$
F_{i}(N)=\sum_{k_{1}=l_{1}}^{L_{1}(N)} \ldots \sum_{k_{v}=l_{v}}^{L_{v}\left(N, k_{1}, \ldots, k_{v-1}\right)} f_{i}\left(N, k_{1}, \ldots, k_{v}\right)
$$

Note that exactly at this point our symbolic summation toolbox in form of the Mathematica package EvaluateMultisums [1, 39] (based on the difference field/ring algorithms in Sigma) can be activated: it tries to transform the found multi-sums completely automatically to the desired form in terms of indefinite nested sums and products. If infinite summation bounds occur one needs 
in addition the Mathematica package HarmonicSums [3, 6, 7] which provides the necessary asymptotic expansions to treat limit computations.

Tactic 2: A different approach is as follows. Hunt for a recurrence

$$
\begin{aligned}
a_{0}(\varepsilon, N) F(N)+a_{1}(\varepsilon, N) F(N+1)+\cdots & +a_{d}(\varepsilon, N) F(N+d) \\
& =h_{\lambda}(N) \varepsilon^{\lambda}+h_{\lambda+1}(N) \varepsilon^{\lambda+1}+h_{\lambda+2}(N) \varepsilon^{\lambda+2}+\ldots
\end{aligned}
$$

of order $d \in \mathbb{N}$ with polynomials $a_{i}(\varepsilon, N)$ in the variables $\varepsilon$ and $N$. In particular, we require that the inhomogeneous part is given in expanded form where the coefficients $h_{i}(N)$ are expressions in terms of indefinite nested sums and products. For the different methods and algorithms to compute such recurrences we refer to [17, 5] and references therein. Here we emphasize the following [17]: Given such a recurrence and suppose that we are given the initial values $F(n)$ for $n=1, \ldots, d$ expanded high enough, then we are in business to obtain the all-N solution using Sigma: we can determine the first coefficients of the expansion (1.3) whenever they are expressible in terms of indefinite nested sums and products.

These tactics (in particular the first variant with EvaluateMultisums) turned out to be instrumental to evaluate two and three loop massive integrals in [15, 2, 那. Another interesting feature is to crunch the occurring sums with the Mathematica package SumProduction [19] to basis sums (master sums) such that no relations (in particular, no contiguous relations) occur among them. Then our summation tools are only applied to a few remaining sums. This enabled us to handle many additional problems such as outlined in Refs. [18, 10] and is currently used for ongoing calculations. For the interplay of all these packages and their features we refer to [41].

For our current calculations, we continue this strategy of compactification by another component. Namely, in order to calculate massive 3-loop operator matrix elements [8, 8, 13] we used integration by parts (IBP) technology [44, 22, 27], more precisely, the powerful $\mathrm{C}++-$ code Reduze 2 [43, 29] was used, to reduce the input expression to a reasonable number of master integrals. Then using our symbolic summation tools (Tactic 1), we could expand the given master integrals in terms of harmonic sums. Given these building blocks we could derive the expansion of the complete input expression.

However, in the most recent calculations integrals occur that seem too hard for direct calculations. This pushed us forward to another aspect. We use the well known fact that the master integrals are related to each other [28]: together they form a hierarchically ordered coupled system of differential (resp. difference) equations.

This article provides a new component to utilize this property by means of symbolic summation (Section 2) and uncoupling algorithms [25]. In Section 3 we will present an algorithm that solves such coupled differential equations in one continuous variable $x$ (resp. coupled difference equations in a discrete variable $N$ ). More precisely, we can derive the first coefficients of the $\varepsilon$ expansion of the master integrals using as input this coupled system and a certain amount of initial values. A summary will be given in Section 0 . 


\section{The backbone of our solver: difference field/ring algorithms}

Subsequently, we work out the essential summation paradigms to treat the two tactics presented above. The underlying ideas will be demonstrated by tackling the following sum

$$
F(N)=\sum_{k=1}^{N} \underbrace{(-1)^{k} e^{-\frac{3 \varepsilon \gamma}{2}} \Gamma\left(-1-\frac{3 \varepsilon}{2}\right) B\left(2+k, \frac{\varepsilon}{2}\right) B(-\varepsilon+k,-\varepsilon) B\left(1-\frac{\varepsilon}{2}+k, 1+\frac{\varepsilon}{2}\right)\left(\begin{array}{l}
N \\
k
\end{array}\right)}_{f(N, k)}
$$

with Euler's $\gamma$ constant and where $B(a, b)=\frac{\Gamma(a) \Gamma(b)}{\Gamma(a+b)}$ denotes the Beta-function.

\subsection{Tactic 1: Expand under the summation sign and simplify the coefficients}

As worked out in the introduction, we first compute the first coefficients $f_{i}(N, k)$ of the $\varepsilon$ expansion (1.4) of the summand $f(N, k)$; here we have $\lambda=-3$. Then we get the coefficients $F_{i}(N)=\sum_{k=1}^{N} f_{i}(N, k)$ of the $\varepsilon$-expansion 1.4). E.g., for $i=-1$ we get the single pole term

$$
F_{-1}(N)=\sum_{k=1}^{N}(-1)^{k+1}\left(\begin{array}{l}
N \\
k
\end{array}\right)\left(\frac{(2+3 k)\left(-2+3 k+7 k^{2}+3 k^{3}\right)}{3 k^{2}(1+k)^{3}}+\frac{2 S_{2}(k)}{1+k}+\frac{\zeta_{2}}{2(1+k)}\right)
$$

with $\zeta_{a}=\sum_{i=1}^{\infty} \frac{1}{i^{a}}$. In order to simplify this sum we compute a recurrence relation using the summation package Sigma:

$$
\begin{aligned}
& \left(16 N^{3}+144 N^{2}+413 N+384\right)(N+1)^{2} F_{-1}(N)-(N+2)(2 N+5)\left(16 N^{3}+112 N^{2}+221 N+113\right) F_{-1}(N+1) \\
& +(N+3)^{2}\left(16 N^{3}+96 N^{2}+173 N+99\right) F_{-1}(N+2)=\frac{\zeta_{2}\left(4 N^{2}+21 N+29\right)}{2}+\frac{-64 N^{5}-500 N^{4}-1133 N^{3}+203 N^{2}+3516 N+3090}{3(N+2)(N+3)} .
\end{aligned}
$$

Remark. The underlying difference field algorithms [32, 35, 36, 37, 40] are based on Zeilberger's creative telescoping paradigm [49]. In general, the input is a definite sum $\sum_{k} f(N, k)$ where the summand $f$ may consist of indefinite nested sums and products w.r.t. the summation variable $k$ and where the occurring objects in $f$ might depend on the parameter $N$ (or even further parameters). Note that the algorithms provide also a proof certificate that shows the correctness of the recurrence.

Now we activate Sigma's recurrence solver, which can handle the following problem [31, 14, 32].

Problem REC: GIVEN polynomials $a_{0}(N), \ldots, a_{d}(N)$ in $N$ and an expression $h(N)$ in terms of indefinite nested sums and products (such as harmonic sums, binomial nested sums, etc.).

FIND all solutions of the linear recurrence

$$
a_{0}(N) F(N)+\cdots+a_{d}(N) F(N+d)=h(N)
$$

that are expressible in terms of indefinite nested sums and products.

In our particular instance, we find the solutions

$$
\begin{aligned}
L=\{ & c_{1} \frac{1-4 N}{N+1}+c_{2}\left(\frac{-14 N-13}{(N+1)^{2}}+\frac{(4 N-1) S_{1}(N)}{N+1}\right)+\frac{(1-4 N) S_{1}(N)^{2}}{6(N+1)} \\
& \left.+\frac{(14 N+13) S_{1}(N)}{3(N+1)^{2}}+\frac{175 N^{2}+334 N+155}{12(N+1)^{3}}+\frac{(1-4 N) S_{2}(N)}{6(N+1)}+\frac{\zeta_{2}}{8(N+1)} \mid c_{1}, c_{2} \in \mathbb{R}\right\} .
\end{aligned}
$$


Since the solution set is completely determined (note that we found two linearly independent solutions of the homogeneous version and one particular solution of the recurrence itself), it follows that $F_{-1}(N) \in L$. The first two initial values $N=1,2$ determine uniquely $c_{1}=\frac{1}{12}-\frac{\zeta_{2}}{8}$ and $c_{2}=1$. Summarizing, we discovered (together with a rigorous proof) that

$$
\begin{aligned}
F_{-1}(N)= & \left(\frac{1}{12}-\frac{1}{8} \zeta_{2}\right) \frac{1-4 N}{N+1}+\frac{-14 N-13}{(N+1)^{2}}+\frac{(4 N-1) S_{1}(N)}{N+1}+\frac{(1-4 N) S_{1}(N)^{2}}{6(N+1)} \\
& +\frac{(14 N+13) S_{1}(N)}{3(N+1)^{2}}+\frac{175 N^{2}+334 N+155}{12(N+1)^{3}}+\frac{(1-4 N) S_{2}(N)}{6(N+1)}+\frac{\zeta_{2}}{8(N+1)} .
\end{aligned}
$$

We remark that the package EvaluateMultisums combines all the available features of Sigma yielding a powerful function to obtain such simplifications in terms of indefinite nested sums and products completely automatically.

\subsection{Tactic 2: Extract the expansion from a recurrence}

For the second tactic we need a recurrence (1.5) for our sum (2.1). Using Sigma we get

$$
\begin{aligned}
& 2(N+1)^{2} F(N)+\left(3 \varepsilon^{2}+3 \varepsilon N+9 \varepsilon-4 N^{2}-12 N-8\right) F(N+1) \\
& \quad-(2 \varepsilon-N-1)(\varepsilon+2 N+6) F(N+2)=0 \varepsilon^{-3}-\frac{16}{3} \varepsilon^{-2}+\frac{40}{3} \varepsilon^{-1}-\left(2 \zeta_{2}-\frac{68}{3}\right) \varepsilon^{0}+\ldots
\end{aligned}
$$

Together with initial values for $N=1$ and $N=2$

$$
F(1)=\frac{2}{3} \varepsilon^{-3}-\frac{11}{6} \varepsilon^{-2}+\left(\frac{\zeta_{2}}{4}+\frac{79}{24}\right) \varepsilon^{-1}+\ldots, \quad F(2)=\frac{8}{9} \varepsilon^{-3}-\frac{73}{27} \varepsilon^{-2}+\left(\frac{\zeta_{2}}{3}+\frac{1415}{324}\right) \varepsilon^{-1}+\ldots
$$

we are now in the position to calculate the first coefficients of the $\varepsilon$-expansion with Sigma:

$$
\begin{aligned}
& F(N)=\frac{4 N}{3(N+1)} \varepsilon^{-3}-\left(\frac{2(2 N+1)}{3(N+1)} S_{1}(N)+\frac{2 N(2 N+3)}{3(N+1)^{2}}\right) \varepsilon^{-2} \\
& \quad\left(\frac{(1-4 N)}{6(N+1)} S_{1}(N)^{2}-\frac{N\left(N^{2}-2\right)}{3(N+1)^{3}}+\frac{(3 N+2)(4 N+5)}{3(N+1)^{2}} S_{1}(N)+\frac{(1-4 N)}{6(N+1)} S_{2}(N)+\frac{N \zeta_{2}}{2(N+1)}\right) \varepsilon^{-1}+\ldots
\end{aligned}
$$

In general, suppose we are given a recurrence (1.5) with polynomial coefficients $a_{i}(N)$ (not all $a_{i}$ being the zero-polynomial) and expressions $h_{i}(N)$ in terms of indefinite nested sums and products; furthermore assume we are given the expansion of $F(i)$ for $i=1, \ldots, d$ up the the order $\varepsilon^{r}$. Then we can decide if the coefficients of the expansion (1.3) up to order $r$ can be expressed in terms of indefinite nested sums and products.

Here the general idea is as follows. Plug in the generic solution (1.3) into (1.5):

$$
\begin{aligned}
& a_{0}(\varepsilon, N)\left[F_{\lambda}(N) \varepsilon^{\lambda}+F_{\lambda+1}(N) \varepsilon^{\lambda+1}+F_{\lambda+2}(N) \varepsilon^{\lambda+2} \ldots\right] \\
+ & a_{1}(\varepsilon, N)\left[F_{\lambda}(N+1) \varepsilon^{\lambda}+F_{\lambda+1}(N+1) \varepsilon^{\lambda+1}+F_{\lambda+2}(N+1) \varepsilon^{\lambda+2}\right]+\cdots+ \\
+ & a_{d}(\varepsilon, N)\left[F_{\lambda}(N+d) \varepsilon^{\lambda}+F_{\lambda+1}(N+d) \varepsilon^{\lambda+1}+F_{\lambda+2}(N+d) \varepsilon^{\lambda+2}+\ldots\right] \\
& =h_{\lambda}(N) \varepsilon^{\lambda}+h_{\lambda+1}(N) \varepsilon^{\lambda+1}+h_{\lambda+2}(N) \varepsilon^{\lambda+2}+\ldots
\end{aligned}
$$

Two Laurent series agree if they agree term-wise, in particular the term with lowest order must agree. This gives the constraint ${ }^{1}$

$$
a_{0}(0, N) F_{\lambda}(N)+a_{1}(0, N) F_{\lambda}(N+1)+\cdots+a_{d}(0, N) F_{\lambda}(N+d)=h_{\lambda}(N) .
$$

\footnotetext{
${ }^{1}$ We auppose that $a_{i}(0, N) \neq 0$ for all $i$; otherwise divide through $\varepsilon$ several times which amounts to decrease $\lambda$.
} 
Now we activate the recurrence solver (see Problem REC on page $\bigoplus$ ) and calculate with Sigma all solutions that are expressible in terms of indefinite nested sums and products. Thus together with the initial values for $F_{\lambda}(1), \ldots, F_{\lambda}(d)$ we can decide if $F_{\lambda}(N)$ can be expressed in terms of indefinite nested sums and products. If this fails, our algorithm stops. Otherwise, we take the found representation of $F_{\lambda}(N)$ in terms of indefinite nested sums and products and plug it into (2.3). Shuffling the inserted expressions to the right hand side gives

$$
\begin{aligned}
& a_{0}(\varepsilon, N)\left[F_{\lambda+1}(N) \varepsilon^{\lambda+1}+F_{\lambda+2}(N) \varepsilon^{\lambda+2} \ldots\right] \\
+ & a_{1}(\varepsilon, N)\left[F_{\lambda+1}(N+1) \varepsilon^{\lambda+1}+F_{\lambda+2}(N+1) \varepsilon^{\lambda+2}\right]+\cdots+ \\
+ & a_{d}(\varepsilon, N)\left[F_{\lambda+1}(N+d) \varepsilon^{\lambda+1}+F_{\lambda+2}(N+d) \varepsilon^{\lambda+2}+\ldots\right]=h_{\lambda+1}^{\prime}(N) \varepsilon^{\lambda+1}+h_{\lambda+2}^{\prime}(N) \varepsilon^{\lambda+2}+\ldots
\end{aligned}
$$

where the $h_{i}^{\prime}(N)$ are updated expressions in terms of indefinite nested sums and products. By construction the $\varepsilon^{\lambda}$ contribution is removed and we can divide the whole equation by $\varepsilon$. Thus we can repeat the procedure where $F_{\lambda+1}$ (instead of $F_{\lambda}$ ) plays the role of the lowest term.

\section{A challenging diagram and a new algorithm to solve coupled systems}

We want to calculate the $\varepsilon$-expansion of the ladder graph with 6 massive fermion lines ${ }^{2}$ :

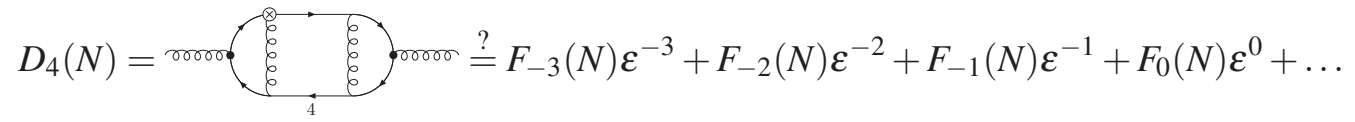

For scalar diagrams of the same class [ [ Tactic 1 of Section 2 But for the diagram in question we failed with this tactic so far. As it turned out, a clever extension [12] of Brown's hyperlogarithm algorithm [21] was successful to obtain in [4] the scalar version of (3.1), i.e. for diagrams with a numerator function equal to one, with $\lim _{\varepsilon \rightarrow 0} D_{4}(N)=F_{0}(N)$. However, if one wants to consider the complete physical diagram, this method does not apply since poles in $\varepsilon$ occur in almost all integrals. Subsequently, we present a new strategy that can tackle such diagrams in a rather natural way.

Let us consider the generating function (formal power series) of $D_{4}(N)$, i.e.,

$$
\hat{D}_{4}(x)=\sum_{N=0}^{\infty} D_{4}(N) x^{N}
$$

Then by using refined IBP methods, i.e., by Reduze 2 [43, 29, 8] we obtain the expression

$$
\sum_{N=0}^{\infty} D_{4}(N) x^{N}=\sum_{i=1}^{52} \square \hat{B}_{i}(x)+\sum_{i=1}^{15} \square \hat{I}_{1}(x)
$$

with the master integrals $\hat{I}_{i}$ and $\hat{B}_{i}$ and with large coefficients $\square$ in terms of rational functions in $\varepsilon$ and $x$ that are not printed out explicitly. As it turns out, $\hat{B}_{1}(x), \ldots, \hat{B}_{52}(x)$ can be determined directly with sophisticated Mellin-Barnes techniques and our summation tools from above. E.g., for

\footnotetext{
${ }^{2}$ The following graph has been drawn using Axodraw [45].
} 
$\hat{B}_{1}(x)=\sum_{N=0}^{\infty} B_{1}(N) x^{N}$ the integral $B_{1}(N)$ can be written in the compact sum representation (2.1) for which we carried out the $\varepsilon$-expansion as a concrete example.

Since $\hat{I}_{1}(x), \ldots, \hat{I}_{15}(x)$ are hard to handle with this toolbox, we use the additional property that the missing integrals satisfy a hierarchically ordered coupled system of differential equations. This particular property is induced by the underlying sector decomposition of the IBP method. More precisely, we are given

$$
\begin{aligned}
D_{x} \hat{I}_{1}(x)= & -\frac{(-\varepsilon+x-1)}{(x-1) x} \hat{I}_{1}(x)-\frac{2}{(x-1) x} \hat{I}_{2}(x)+\frac{1}{(x-1) x} \hat{B}_{1}(x)+\ldots \\
D_{x} \hat{I}_{2}(x)= & -\frac{\varepsilon(3 \varepsilon+2)(x-2)}{4(x-1) x} \hat{I}_{1}(x)+\frac{(-2+x+\varepsilon(3 x-5))}{2(x-1) x} \hat{I}_{2}(x)-\frac{(2 \varepsilon+x-\varepsilon x)}{2(x-1) x} \hat{I}_{3}(x) \\
& +\frac{\varepsilon(50-14 x)+\varepsilon^{2}(25-6 x)-8(x-3)}{4(5 \varepsilon+6)(x-1) x} \hat{B}_{1}(x)+\ldots \\
D_{x} \hat{I}_{3}(x)= & \frac{\varepsilon(3 \varepsilon+2)}{4(x-1)} \hat{I}_{1}(x)+\frac{(2+\varepsilon-3 x-3 \varepsilon x)}{2(x-1) x} \hat{I}_{2}(x)-\frac{(\varepsilon+1)}{2(x-1)} \hat{I}_{3}(x)+\frac{8(x-3)+\varepsilon^{2}(6 x-25)+2 \varepsilon(7 x-25)}{4(5 \varepsilon+6)(x-1) x} \hat{B}_{1}(x)
\end{aligned}
$$

in terms of $\hat{I}_{1}(x), \hat{I}_{2}(x), \hat{I}_{3}(x)$. Note that by the internal structure the right hand sides of (3.3) are free of $\hat{I}_{4}(x), \ldots, \hat{I}_{15}(x)$. In the following subsections we will demonstrate how this system can be solved in $\hat{I}_{1}(x), \hat{I}_{2}(x), \hat{I}_{3}(x)$ by using the explicitly given expansions of the $\hat{B}_{i}$ that we calculated already as a preprocessing step.

Given this result, we will then turn to the remaining $\hat{I}_{i}(x)$ with $i>3$. Here the hierarchical nature proceeds. Given the $\varepsilon$-expansion of the $\hat{B}_{i}(x)$ and $\hat{I}_{1}(x), \hat{I}_{2}(x), \hat{I}_{3}(x)$ in terms of indefinite nested sums and products, we obtain a coupled system in terms of the unknowns $\left\{\hat{I}_{4}(x), \hat{I}_{5}(x)\right\}$ and we can solve them again in terms of indefinite nested sums and products. Summarizing, we continue iteratively, and obtain closed forms for the clustered integrals in the given hierarchically structured order:

$$
\begin{aligned}
\left\{\hat{I}_{1}(x), \hat{I}_{2}(x), \hat{I}_{3}(x)\right\} \rightarrow & \left\{\hat{I}_{4}(x), \hat{I}_{5}(x)\right\} \rightarrow\left\{\hat{I}_{6}(x), \hat{I}_{7}(x), \hat{I}_{8}(x)\right\} \\
& \rightarrow\left\{\hat{I}_{9}(x), \hat{I}_{10}(x)\right\} \rightarrow\left\{\hat{I}_{11}(x), \hat{I}_{12}(x), \hat{I}_{13}(x)\right\} \rightarrow\left\{\hat{I}_{14}(x)\right\} \rightarrow\left\{\hat{I}_{15}(x)\right\} .
\end{aligned}
$$

\subsection{Step 1: Transformation to a coupled recurrence system}

In order to solve the coupled system (3.3), we first derive a coupled system of difference equations that determines the coefficients $I_{1}(N), I_{2}(N), I_{3}(N)$ of the power series

$$
\hat{I}_{i}(x)=\sum_{N=0}^{\infty} I_{i}(N)
$$

Namely, plugging in these generating functions into the first equation of (3.3) yields

$$
D_{x} \sum_{N=0}^{\infty} I_{1}(N) x^{N}=-\frac{(-\varepsilon+x-1)}{(x-1) x} \sum_{N=0}^{\infty} I_{1}(N) x^{N}-\frac{2}{(x-1) x} \sum_{N=0}^{\infty} I_{2}(N) x^{N}+\frac{1}{(x-1) x} \sum_{N=0}^{\infty} B_{1}(N) x^{N}+\ldots
$$

Then applying $D_{x}$ on the summands of the power series and doing coefficient comparison on both sides leads to

$$
N I_{1}(N-1)-(\varepsilon+N+1) I_{1}(N)+2 I_{2}(N)=B_{1}(N)+\ldots,
$$

where on the right hand sides only the master integrals $B_{i}(N)$ (but not $I_{i}$ ) arise. Similarly, we apply this transformation to the other equations in (3.3) and inserting the already computed $\varepsilon$ - expansions 
of $B_{i}(N)$ we obtain the coupled recurrence system

$$
\begin{aligned}
N I_{1}(N-1) & -(\varepsilon+N+1) I_{1}(N)+2 I_{2}(N) \\
= & -\frac{4(N+2)}{3(N+1)} \varepsilon^{-3}+\left(\frac{2(2 N+1)}{3(N+1)} S_{1}(N)-\frac{2\left(6 N^{2}+13 N+8\right)}{3(N+1)^{2}}\right) \varepsilon^{-2}+\ldots \\
4(\varepsilon-N) I_{3}(N)-2 \varepsilon(3 \varepsilon+2) I_{1}(N)+\varepsilon(3 \varepsilon+2) I_{1}(N-1) & \\
& \quad 2(3 \varepsilon+1) I_{2}(N-1)+2(5 \varepsilon+2) I_{2}(N)-2(\varepsilon-2 N+1) I_{3}(N-1) \\
= & -\frac{8}{3} \varepsilon^{-3}-\left(\frac{8}{3} S_{1}(N)-4\right) \varepsilon^{-2}-\left(\frac{4}{3} S_{1}(N)^{2}-\frac{4(N+1)}{N} S_{1}(N)+\frac{4}{3} S_{2}(N)+\zeta_{2}+6\right) \varepsilon^{-1}+\ldots \\
2(\varepsilon+ & 2 N+2) I_{2}(N)-2(3 \varepsilon+2 N+1) I_{2}(N-1)+\varepsilon(3 \varepsilon+2) I_{1}(N-1)-2(\varepsilon+1) I_{3}(N-1) \\
= & \frac{8}{3} \varepsilon^{-3}+\left(\frac{8}{3} S_{1}(N)-4\right) \varepsilon^{-2}+\left(\frac{4}{3} S_{1}(N)^{2}-\frac{4(N+1)}{N} S_{1}(N)+\frac{4}{3} S_{2}(N)+\zeta_{2}+6\right) \varepsilon^{-1}+\ldots,
\end{aligned}
$$

where the left hand sides contain the unknowns $I_{1}(N), I_{2}(N), I_{3}(N)$ and the right hand sides consist of $\varepsilon$-expansions whose coefficients are given in terms of indefinite nested sums and products. More precisely, in our concrete example, only harmonic sums occur.

\subsection{Step 2: Uncouple the recurrence system}

In the next step we uncouple the system (3.5) in the following sense: we search for one scalar linear recurrence in one of the functions, say $I_{1}(N)$, and express the remaining functions $I_{2}(N)$ and $I_{3}(N)$ in terms of $I_{1}(N)$. To accomplish this task, various algorithms are available within the Mathematica package OreSys [25]; for our concrete problem we took Zürcher's uncoupling algorithm [50].

More precisely, we get the scalar difference equation

$$
\begin{gathered}
-2(N+1)(N+2)(\varepsilon+N+2) I_{1}(N)-(N+2)\left(2 \varepsilon^{2}-5 \varepsilon N-7 \varepsilon-6 N^{2}-28 N-32\right) I_{1}(N+1) \\
+\left(\varepsilon^{3}+4 \varepsilon^{2} N+14 \varepsilon^{2}-4 \varepsilon N^{2}-13 \varepsilon N-3 \varepsilon-6 N^{3}-50 N^{2}-136 N-120\right) I_{1}(N+2) \\
-(\varepsilon-N-2)(\varepsilon+N+4)(\varepsilon+2 N+8) I_{1}(N+3) \\
=-\frac{4(N+2)}{3(N+3)} \varepsilon^{-3}+\frac{2\left(4 N^{4}+35 N^{3}+101 N^{2}+105 N+25\right)}{3(N+1)(N+2)(N+3)^{2}} \varepsilon^{-2}+\ldots
\end{gathered}
$$

in the unknown function $I_{1}(N)$ and the two equations

$$
\begin{aligned}
I_{2}(N)= & \square I_{1}(N)+\square I_{1}(N+1)+\square I_{1}(N+2) \\
& -\frac{2(N+2)}{3(N+1)} \varepsilon^{-3}+\left(\frac{6 N^{3}+25 N^{2}+33 N+15}{3(N+1)^{2}(N+2)}+\frac{(-2 N-1)}{3(N+1)} S_{1}(N)\right) \varepsilon^{-2}+\ldots \\
I_{3}(N)= & \square I_{1}(N)+\square I_{1}(N+1)+\square I_{1}(N+2) \\
& +\frac{2(N+2)}{3(N+1)} \varepsilon^{-3}+\left(\frac{-2 N^{3}-3 N^{2}+3 N+3}{3(N+1)^{2}(N+2)}+\frac{(2 N+1)}{3(N+1)} S_{1}(N)\right) \varepsilon^{-2}+\ldots
\end{aligned}
$$

that determine $I_{2}(N)$ and $I_{3}(N)$ if one knows the solution of $I_{1}(N)$.

\subsection{Step 3: Solve the uncoupled system of difference equations}

Now we are in the right position to activate Tactic 2 of our symbolic summation toolbox. First we derive the initial values

$$
\begin{aligned}
& I_{1}(1)=\frac{5}{\varepsilon^{3}}-\frac{163}{12 \varepsilon^{2}}+\left(\frac{15 \zeta_{2}}{8}+\frac{1223}{48}\right) \varepsilon^{-1}+\ldots, \quad I_{1}(2)=\frac{130}{27 \varepsilon^{3}}-\frac{695}{54 \varepsilon^{2}}+\left(\frac{65 \zeta_{2}}{36}+\frac{46379}{1944}\right) \varepsilon^{-1}+\ldots, \\
& I_{1}(3)=\frac{169}{36 \varepsilon^{3}}-\frac{395}{32 \varepsilon^{2}}+\left(\frac{169 \zeta_{2}}{96}+\frac{470071}{20736}\right) \varepsilon^{-1}+\ldots
\end{aligned}
$$


using, e.g., MATAD [42] or using further tools as Mellin-Barnes integrals and other methods as worked out in [10]. Namely, given (3.6) we activate Sigma's recurrence solver and obtain the $\varepsilon$-expansion

$$
\begin{aligned}
I_{1}(N) & =\left(\frac{4\left(3 N^{2}+6 N+4\right)}{3(N+1)^{2}}+\frac{4 S_{1}(N)}{3(N+1)}\right) \varepsilon^{-3} \\
& +\left(\frac{-2\left(20 N^{3}+58 N^{2}+57 N+22\right)}{3(N+1)^{3}}-\frac{S_{1}(N)^{2}}{N+1}+\frac{2(N+2)(2 N-1) S_{1}(N)}{3(N+1)^{2}}-\frac{S_{2}(N)}{N+1}\right) \varepsilon^{-2}+\ldots
\end{aligned}
$$

where the coefficients are given in terms of harmonic sums. Finally, we utilize (3.7) and get

$$
\begin{aligned}
& I_{2}(N)=\frac{4}{3} \varepsilon^{-3}-\frac{2}{\varepsilon^{2}}+\left(-\frac{1}{3} S_{1}(N)^{2}+\frac{2}{3} S_{1}(N)-\frac{1}{3} S_{2}(N)+\frac{5 N+7}{3(N+1)}+\frac{\zeta_{2}}{2}\right) \varepsilon^{-1}+\ldots \\
& I_{3}(N)=-\frac{8}{3 \varepsilon^{3}}+\left(\frac{4(N+2)}{3(N+1)} S_{1}(N)-\frac{4\left(4 N^{2}+7 N+2\right)}{3(N+1)^{2}}\right) \varepsilon^{-2} \\
& \quad+\left(\frac{2\left(12 N^{3}+32 N^{2}+25 N+2\right)}{3(N+1)^{3}}-\frac{2\left(4 N^{2}+11 N+10\right)}{3(N+1)^{2}} S_{1}(N)+\frac{(N-2)}{3(N+1)} S_{1}(N)^{2}+\frac{(N-2)}{3(N+1)} S_{2}(N)+\zeta_{2}\right) \varepsilon^{-1}+\ldots
\end{aligned}
$$

\subsection{The general method and the physical result of $D_{4}(N)$}

Summarizing, we calculated the first coefficients of the $\varepsilon$-expansions of $\hat{I}_{1}(N), \hat{I}_{2}(N), \hat{I}_{3}(N)$ (resp. of $I_{1}(N), I_{2}(N), I_{3}(N)$ ) and treat also all other integrals in $(3.4)$ iteratively by the following method.

Step 1: Transform the coupled DE system to a coupled REC System $(x \rightarrow N)$.

Step 2: Uncouple the REC system to a scalar recurrence for one unknown integral, say $I_{i}(N)$.

Step 3: Determine the coefficients of the $\varepsilon$-expansion of $I_{i}(N)$ in terms of indefinite nested sums and products (see Tactic 2 in Section 2 ) and derive the $\varepsilon$-expansions of the remaining integrals.

Step 4: Translate back to the $x$-space by $\hat{I}_{i}(x)=\sum_{N=0}^{\infty} I_{i}(N) x^{N}$.

To this end, we plug in all the computed expansions $\hat{B}_{i}(x)$ (by using symbolic summation) and $\hat{I}_{i}(x)$ (by using our new solver for coupled equations) into the expression (3.2). This actually gives again a gigantic expression in terms of generating functions where the coefficients of the $\varepsilon$-expansion are extremely large. In order to derive the $N$ th coefficient $D_{4}(N)$, we activate again our toolbox mentioned in the introduction. We crunch the arising generating functions with the package SumProduction and compute the $N$ th coefficient of the obtained compact expression using the package HarmonicSums. Finally, observe that this operation is based on Cauchyproduct and we therefore obtain again definite sums. Finally, we apply once more Tactic 1 of our symbolic summation toolbox, more precisely we use the package EvaluateMultiSums based on Sigma to transform these sums to indefinite nested sums and products. 
Summarizing, using all these packages, we end up at the following result for (3.1):

$$
\begin{aligned}
D_{4}(N)= & \left(\frac{64\left(N^{2}+N-1\right)}{3(N+1)(N+2)(N+3)(N+4)}-\frac{64 S_{1}(N)}{3(N+3)(N+4)}\right) \varepsilon^{-3} \\
& +\left(\frac{4(N+1)(4 N+17) S_{2}(N)}{3(N+2)(N+3)(N+4)}-\frac{4\left(3 N^{5}+68 N^{4}+379 N^{3}+648 N^{2}-98 N-696\right)}{3(N+1)(N+2)^{2}(N+3)^{2}(N+4)^{2}} S_{1}(N)\right. \\
& +\frac{4\left(14 N^{6}+214 N^{5}+1179 N^{4}+3050 N^{3}+4097 N^{2}+3094 N+1200\right)}{3(N+1)^{2}(N+2)^{2}(N+3)^{2}(N+4)^{2}} \\
& \left.+\frac{4(5 N+27)}{3(N+2)(N+3)(N+4)} S_{1}(N)^{2}\right) \varepsilon^{-2}+\ldots
\end{aligned}
$$

The single pole term and constant term are suppressed due to space limitations. In total the following harmonic sums and generalized harmonic sums occur

$$
\begin{aligned}
& \zeta_{2}, \zeta_{3},(-1)^{N}, 2^{N}, S_{-3}(N), S_{1}(N), S_{2}(N), S_{3}(N), S_{4}(N), S_{-2,1}(N), S_{2,1}(N), S_{3,1}(N), \\
& S_{1}\left(\frac{1}{2}, N\right), S_{1}(2, N), S_{3}\left(\frac{1}{2}, N\right), S_{1,1}\left(1, \frac{1}{2}, N\right), S_{1,1}\left(2, \frac{1}{2}, N\right), S_{2,1,1}(N), S_{2,1}\left(\frac{1}{2}, 1, N\right), \\
& S_{2,1}\left(1, \frac{1}{2}, N\right), S_{3,1}\left(\frac{1}{2}, 2, N\right), S_{1,1,1}\left(1,1, \frac{1}{2}, N\right), S_{2,1,1}\left(1, \frac{1}{2}, 2, N\right), S_{1,1,1,1}\left(2, \frac{1}{2}, 1,1, N\right) .
\end{aligned}
$$

\section{Conclusion}

We presented a new method to solve coupled systems of differential and difference equations which emerge in massive Feynman diagram calculations. Here we rely on sophisticated summation tools based on difference fields/rings and on uncoupling algorithms; for our concrete example we used the package OreSys [25].

We obtained the $\varepsilon$-expansions of rather complicated master integrals. Using these expansions we calculated easily the most complicated ladder graphs with 6 massive fermion lines using Sigma HarmonicSums, EvaluateMultiSums and SumProduction. All ladder-topologies for 3loop massive operator matrix elements can be calculated in this way. The mass production is ready for graphs depending on the same master integrals. We used this technology for a few integrals emerging in Feynman integrals with two equal masses [10] and in the pure-singlet case [13]. More involved massive 3-loop topologies are currently investigated.

\section{References}

[1] J. Ablinger, J. Blümlein, S. Klein and C. Schneider, Modern Summation Methods and the Computation of 2- and 3-loop Feynman Diagrams, Nucl. Phys. Proc. Suppl. 205-206 (2010) 110-115 [arXiv:1006.4797 [math-ph]].

[2] J. Ablinger, J. Blümlein, S. Klein, C. Schneider and F. Wißbrock, The $O\left(\alpha_{s}^{3}\right)$ Massive Operator Matrix Elements of $O\left(N_{f}\right)$ for the Structure Function $F_{2}\left(x, Q^{2}\right)$ and Transversity, Nucl. Phys. B 844 (2011) 26-54 [arXiv:1008.3347 [hep-ph]].

[3] J. Ablinger, J. Blümlein and C. Schneider, Harmonic Sums and Polylogarithms Generated by Cyclotomic Polynomials, J. Math. Phys. 52 (2011) 102301 [arXiv:1105.6063 [math-ph]].

[4] J. Ablinger, J. Blümlein, A. Hasselhuhn, S. Klein, C. Schneider and F. Wißbrock, Massive 3-loop Ladder Diagrams for Quarkonic Local Operator Matrix Elements, Nucl. Phys. B 864 (2012) 52-84 [arXiv:1206.2252 [hep-ph]]. 
[5] J. Ablinger, J. Blümlein, M. Round and C. Schneider, Advanced Computer Algebra Algorithms for the Expansion of Feynman Integrals, PoS LL 2012 (2012) 050 [arXiv:1210.1685 [cs.SC]].

[6] J. Ablinger, Computer Algebra Algorithms for Special Functions in Particle Physics, Ph.D. thesis, RISC, J. Kepler University Linz, 2013 arXiv:1305.0687 [math-ph].

[7] J. Ablinger, J. Blümlein and C. Schneider, Analytic and Algorithmic Aspects of Generalized Harmonic Sums and Polylogarithms, J. Math. Phys. 54 (2013) 082301 [arXiv:1302.0378 [math-ph]].

[8] J. Ablinger, A. Behring, J. Blümlein, A. De Freitas, A. Hasselhuhn, A. von Manteuffel, M. Round and C. Schneider and F. Wißbrock, The 3-Loop Non-Singlet Heavy Flavor Contributions and Anomalous Dimensions for the Structure Function $F_{2}\left(x, Q^{2}\right)$ and Transversity, arXiv:1406.4654 [hep-ph].

[9] J. Ablinger, J. Blümlein, A. De Freitas, A. Hasselhuhn, A. von Manteuffel, M. Round, C. Schneider and F. Wißbrock, The Transition Matrix Element $A_{g q}(N)$ of the Variable Flavor Number Scheme at $O\left(\alpha_{s}^{3}\right)$, Nucl. Phys. B 882 (2014) 263-288 [arXiv:1402.0359 [hep-ph]].

[10] J. Ablinger, J. Blümlein, A. De Freitas, A. Hasselhuhn, A. von Manteuffel, M. Round and C. Schneider, The $O\left(\alpha_{s}^{3} T_{F}^{2}\right)$ Contributions to the Gluonic Operator Matrix Element Nucl. Phys. B 885 (2014) 280-317 [arXiv:1405.4259 [hep-ph]].

[11] J. Ablinger, J. Blümlein, C. G. Raab and C. Schneider, Iterated Binomial Sums and their Associated Iterated Integrals, arXiv:1407.1822 [hep-th].

[12] J. Ablinger, J. Blümlein, C. Raab, C. Schneider and F. Wißbrock, Calculating Massive 3-loop Graphs for Operator Matrix Elements by the Method of Hyperlogarithms, Nucl. Phys. B 885 (2014) 409-447 [arXiv:1403.1137 [hep-ph]].

[13] J. Ablinger et al., DESY 13-232, to be published.

[14] S.A. Abramov and M. Petkovšek, D’Alembertian solutions of linear differential and difference equations, In: J. von zur Gathen (ed.) Proc. ISSAC'94, pp. 169-174 ACM Press (1994).

[15] I. Bierenbaum, J. Blümlein, S. Klein and C. Schneider, Two-Loop Massive Operator Matrix Elements for Unpolarized Heavy Flavor Production to $O(\varepsilon)$, Nucl. Phys. B 803 (2008) 1-41 [arXiv:0803.0273 [hep-ph]].

[16] J. Blümlein and S. Kurth, Harmonic sums and Mellin transforms up to two loop order, Phys. Rev. D 60 (1999) 014018 [arXiv:9810241 [hep-ph]].

[17] J. Blümlein, S. Klein, C. Schneider and F. Stan, A Symbolic Summation Approach to Feynman Integral Calculus, J. Symbolic Comput. 47 (2012) 1267-1289 [arXiv:1011.2656 [cs.SC]].

[18] J. Blümlein, A. Hasselhuhn, S. Klein and C. Schneider, The $O\left(\alpha_{s}^{3} N_{f} T_{F}^{2} C_{A, F}\right)$ Contributions to the Gluonic Massive Operator Matrix Elements, Nucl. Phys. B 866 (2013) 196-211 [arXiv:1205.4184 [hep-ph]].

[19] J. Blümlein, A. Hasselhuhn and C. Schneider, Evaluation of Multi-Sums for Large Scale Problems, PoS RADCOR 2011 (2011) 032 [arXiv:1202.4303 [math-ph]].

[20] M. Bronstein, On solutions of linear ordinary difference equations in their coefficient field, J. Symbolic Comput. 29 (2000) 841-877.

[21] F.C.S. Brown, The Massless higher-loop two-point function, Commun. Math. Phys. 287 (2009) 925-958 [arXiv:0804.1660 [math.AG]].

[22] K. G. Chetyrkin and F. V. Tkachov, Integration by Parts: The Algorithm to Calculate beta Functions in 4 Loops, Nucl. Phys. B 192 (1981) 159-204 
[23] A. I. Davydychev and M. Y. Kalmykov, Massive Feynman diagrams and inverse binomial sums, Nucl. Phys. B 699 (2004) 3-64 [arXiv:0303162 [hep-th]].

[24] J. Fleischer, A. V. Kotikov and O. L. Veretin, Analytic two loop results for selfenergy type and vertex type diagrams with one nonzero mass, Nucl. Phys. B 547 (1999) 343-374 [hep-ph/9808242].

[25] S. Gerhold, Uncoupling systems of linear Ore operator equations, Master's thesis, RISC, J. Kepler University, Linz, 2002.

[26] M. Karr, Summation in finite terms, J. ACM, 28 (1981) 305-350.

[27] S. Laporta and E. Remiddi, The Analytical value of the electron $(g-2)$ at order $\alpha^{3}$ in $Q E D$, Phys. Lett. B 379 (1996) 283-291 [arXiv:9602417 [hep-ph]].

[28] S. Laporta, High precision calculation of multiloop Feynman integrals by difference equations, Int. J. Mod. Phys. A 15 (2000) 5087-5159 [arXiv:0102033 [hep-ph]].

[29] A. von Manteuffel and C. Studerus, Reduze2 - Distributed Feynman Integral Reduction, 2012 [arXiv:1201.4330 [hep-ph]].

[30] S. Moch, P. Uwer and S. Weinzierl, Nested sums, expansion of transcendental functions and multiscale multiloop integrals, J. Math. Phys. 43 (2002) 3363-3386 [arXiv:0110083 [hep-ph]].

[31] M. Petkovšek, Hypergeometric solutions of linear recurrences with polynomial coefficients, J. Symbolic Comput. 14 (1992) 243-264.

[32] C. Schneider, Symbolic Summation in Difference Fields, Ph.D. Thesis RISC, Johannes Kepler University, Linz technical report 01-17 (2001).

[33] C. Schneider, Solving parameterized linear difference equations in terms of indefinite nested sums and products, J. Differ. Equations Appl. 11 (2005) 799-821.

[34] C. Schneider, Symbolic summation assists combinatorics, Sém. Lothar. Combin. 56 (2007) 1-36 article B56b.

[35] C. Schneider, A refined difference field theory for symbolic summation, J. Symbolic Comput. 43 (2008) 611-644 [arXiv:0808.2543 [cs.SC]].

[36] C. Schneider, Structural Theorems for Symbolic Summation, Appl. Algebra Engrg. Comm. Comput. 21 (2010) 1-32.

[37] C. Schneider, A Symbolic Summation Approach to Find Optimal Nested Sum Representations, In A. Carey, D. Ellwood, S. Paycha, and S. Rosenberg, editors, Motives, Quantum Field Theory, and Pseudodifferential Operators, Vol. 12 Clay Mathematics Proceedings, Amer. Math. Soc., pp. 285-308, (2010) [arXiv:0808.2543 [cs.SC]].

[38] C. Schneider, Parameterized Telescoping Proves Algebraic Independence of Sums, Ann. Comb. 14 (2010) 533-552 [arXiv:0808.2596 [cs.SC]].

[39] C. Schneider, Simplifying Multiple Sums in Difference Fields, in: Computer Algebra in Quantum Field Theory: Integration, Summation and Special Functions, Texts \& Monographs in Symbolic Computation eds. C. Schneider and J. Blümlein (Springer, Wien, 2013) 325-360 [arXiv:1304.4134 [cs.SC]].

[40] C. Schneider, Fast Algorithms for Refined Parameterized Telescoping in Difference Fields, in : Lecture Notes in Computer Science (LNCS) eds. J. Guitierrez, J. Schicho, M. Weimann, in press (2014) [arXiv:13077887 [cs.SC]]. 
[41] C. Schneider, Modern Summation Methods for Loop Integrals in Quantum Field Theory: The Packages Sigma, EvaluateMultiSums and SumProduction, J. Phys. Conf. Ser. 523 (2014) 012037 [arXiv:1310.0160 [cs.SC]].

[42] M. Steinhauser, MATAD : A Program package for the computation of MAssive TADpoles, Comput. Phys. Commun. 134 (2001) 335-364 [hep-ph/0009029].

[43] C. Studerus, Reduze-Feynman Integral Reduction in $\mathrm{C}++$, Comput. Phys. Commun. 181 (2010) 1293 [arXiv:0912.2546 [physics.comp-ph]].

[44] F. V. Tkachov, A Theorem on Analytical Calculability of Four Loop Renormalization Group Functions, Phys. Lett. B 100 (1981) 65-68.

[45] J.A.M. Vermaseren, Axodraw, Comput. Phys. Commun. 83 (1994) 45-58.

[46] J.A.M. Vermaseren, Harmonic sums, Mellin transforms and integrals, Int. J. Mod. Phys. A 14 (1999) 2037-2076 [arXiv: 9806280 [hep-ph]].

[47] S. Weinzierl, Expansion around half integer values, binomial sums and inverse binomial sums, J. Math. Phys. 45 (2004) 2656-2673 [arXiv:0402131 [hep-ph]].

[48] S. Weinzierl, Feynman graphs, in: Computer Algebra in Quantum Field Theory: Integration, Summation and Special Functions, Texts \& Monographs in Symbolic Computation eds. C. Schneider and J. Blümlein (Springer, Wien, 2013) 381-406 (2013) [arXiv:13016918 [hep-ph]].

[49] D. Zeilberger, The method of creative telescoping, J. Symbolic Comput. 11, 195-204 (1991).

[50] B. Zürcher, Rationale Normalformen von pseudo-linearen Abbildungen, Master's thesis, Mathematik, ETH Zürich, 1994. 